\title{
Teaching emergency medicine with workshops improved medical student satisfaction in emergency medicine education
}

This article was published in the following Dove Press journal:

Advances in Medical Education and Practice

2 February 2015

Number of times this article has been viewed

\author{
Pungkava Sricharoen' \\ Chaiyaporn Yuksen' \\ Yuwares Sittichanbuncha' \\ Kittisak Sawanyawisuth ${ }^{2,3}$ \\ 'Department of Emergency Medicine, \\ Faculty of Medicine, Ramathibodi \\ Hospital, Mahidol University, Bangkok, \\ Thailand; ${ }^{2}$ Department of Medicine, \\ Faculty of Medicine, Khon Kaen \\ University, Khon Kaen, Thailand; ${ }^{3}$ The \\ Research Center in Back, Neck, Other \\ Joint Pain and Human Performance \\ (BNOJPH), Khon Kaen University, \\ Khon Kaen, Thailand
}

Background: There are different teaching methods; such as traditional lectures, bedside teaching, and workshops for clinical medical clerkships. Each method has advantages and disadvantages in different situations. Emergency Medicine (EM) focuses on emergency medical conditions and deals with several emergency procedures. This study aimed to compare traditional teaching methods with teaching methods involving workshops in the EM setting for medical students.

Methods: Fifth year medical students (academic year of 2010) at Ramathibodi Hospital, Faculty of Medicine, Mahidol University, Bangkok, Thailand participated in the study. Half of students received traditional teaching, including lectures and bedside teaching, while the other half received traditional teaching plus three workshops, namely, airway workshop, trauma workshop, and emergency medical services workshop. Student evaluations at the end of the clerkship were recorded. The evaluation form included overall satisfaction, satisfaction in overall teaching methods, and satisfaction in each teaching method.

Results: During the academic year 2010, there were 189 students who attended the EM rotation. Of those, 77 students $(40.74 \%)$ were in the traditional EM curriculum, while 112 students were in the new EM curriculum. The average satisfaction score in teaching method of the new EM curriculum group was higher than the traditional EM curriculum group (4.54 versus 4.07, $P$-value $<0.001)$. The top three highest average satisfaction scores in the new EM curriculum group were trauma workshop, bedside teaching, and emergency medical services workshop. The mean (standard deviation) satisfaction scores of those three teaching methods were 4.70 (0.50), 4.63 (0.58), and $4.60(0.55)$, respectively.

Conclusion: Teaching EM with workshops improved student satisfaction in EM education for medical students.

Keywords: emergency medicine education, workshop, student satisfaction

\section{Introduction}

There are several learning methods in medical education. Traditional lectures have some benefits such as being less time consuming for both instructors and students. The main disadvantage of traditional lectures is that students have less time in analytical practice. ${ }^{1} \mathrm{~A}$ workshop is a teaching method that emphasizes student-teacher interaction in real situations. Students have time to think, analyze, and apply their knowledge to the problems they encounter. ${ }^{2,3}$ Bedside teaching is a logical style of teaching in clinical medicine. It stimulates students in clinical thinking, clinical skills, communication skills, and ethical issues with real patients. ${ }^{4-6}$ There are several factors that may affect the outcomes of teaching strategies such as contents, student characteristics, number of students, and cultures. However, the teaching method is one factor that may improve learning processes of students. ${ }^{5}$
Correspondence: Kittisak Sawanyawisuth Department of Medicine, Faculty of Medicine, 123 Mittraphap Road, Khon Kaen University, Khon Kaen, 40002. Thailand

Tel +6643363664

Fax +6643348399

Email kittisak@kku.ac.th (c) (i) (5) 2015 Sricharoen et al. This work is published by Dove Medical Press Limited, and licensed under Creative Commons Attribution - Non Commercial (unported, v3.0) BY LC License. The full terms of the License are available at http://creativecommons.org/licenses/by-nc/3.0/. Non-commercial uses of the work are permitted without any further perminson how to request permission may be found at: http://www.dovepress.com/permissions.php 
Emergency Medicine (EM) curriculum focuses on clinical learning in all emergency conditions that may require several procedures. Clinical skills therefore are an important factor to manage patients appropriately and promptly. Before 2010, EM at Ramathibodi Hospital, Mahidol University had only traditional lectures and bedside teaching for fifth year medical students. We implemented additional workshops to the EM curriculum and evaluated their effects.

\section{Methods}

The traditional EM curriculum comprised of traditional lectures and bedside teaching in common emergency conditions for medical students based on the Thai Medical Council curriculum. The traditional EM curriculum was used until the academic year of 2010. In 2010 mid-year, a new EM curriculum was introduced. The new curriculum comprised of traditional lectures, bedside teaching, and three additional workshops. Teaching hours for traditional lectures and bedside teaching were similar to the traditional EM curriculum in the new EM curriculum. Three workshops lasting about 3 hours each and comprising of airway, trauma, and emergency medical services (EMS) workshops were added. Characteristics of each teaching method are as follows:

- Traditional lecture: one instructor per topic in EM in a lecture hall.

- Bedside teaching: one student had a complete case of EM including history taking, physical examination, laboratory results, and medical report writing under supervision of one preceptor.

- Airway workshop (3 hours and 15 minutes): comprised of two parts; airway management and ventilator support. The contents included descriptions of devices, indications, contraindications, complications, and demonstrations by instructors. Some students participated in the practice of endotracheal/nasotracheal intubation and respiratory settings with a training mannequin.

- Trauma workshop (3 hours): contained brief on trauma case care and examples of trauma situations. Students were divided into small groups of 4-5 and practiced advanced trauma life support in different situations. Preceptors observed and commented on students' practice.

- EMS workshop (2 hours and 30 minutes): teaching about emergency medical service in Thailand including referral system, ambulance, medical devices in ambulance, patient transfer, and patient transfer devices such as spinal board and stair chair. The stair chair is used to move a patient over stairs, while the spinal board is used to move a suspected spinal cord injury patient in case of emergency transfer.

\section{Study population}

The fifth year medical students at Ramathibodi University Hospital, Mahidol University, Thailand in the year 2010 were enrolled. All students gave a blind evaluation at the end of the 3 week EM course. Half of the students were in the traditional EM curriculum group, and the remaining half were in the new EM curriculum.

During the 3 week EM rotation, there were 12 lectures in the morning sessions, teaching round, and ER clerkship (Table 1). For the workshop curriculum, two workshops were scheduled on Friday of the second week and one workshop was scheduled on Wednesday of the third week; the rest of the activities were similar to the traditional curriculum.

The evaluation form was a 5-point Likert scale; 1 referred to least satisfied and 5 referred to most satisfied. Items for evaluation included overall satisfaction, satisfaction in overall teaching methods, and satisfaction in each teaching method. The study protocol was approved by the Institution Research Board, Mahidol University.

\section{Statistical analyses}

Descriptive statistics were used to report satisfaction level in all items by points out of 5 . Student's $t$-test was used to compare score in overall satisfaction, satisfaction in overall teaching methods, and satisfaction in each teaching method between both curriculums. Differences between each teaching method in the new EM curriculum were calculated by repeated measures analysis of variance (ANOVA) with

Table I Teaching schedule for Emergency Department rotation for the fifth year medical students

\begin{tabular}{llll}
\hline Week & 7:45-8:45 & 9:00-10:00 & I 0:00-1 7:00 \\
\hline \multicolumn{1}{l}{ Traditional curriculum } & Teaching round & ER observers \\
I & Lectures & Teaching round & ER observers \\
2 & Lectures & Teaching round & ER observers \\
3 & Lectures & Teaching round & ER observers \\
New Emergency Medicine curriculum & Teaching round & ER observers \\
I & Lectures & & \\
2 & Lectures, two & Teaching round & ER observers \\
& workshops on Friday & & \\
\hline
\end{tabular}

Notes: Twelve lecture topics are: communication and ER management; radiology for ER; treatment of shock; common pediatric injuries; acute chest pain/dyspnea; psychological problems in ER; ventilation support; asthmatic attack; pediatric advanced cardiac life support; antimicrobial agents in life threatening infection; ER management of the injured patients; and acute poisoning.

Abbreviation: ER, emergency room. 
Bonferroni correction. Students who did not complete an evaluation for each teaching method were excluded from the analysis by repeated measures ANOVA. Statistical analyses were executed by STATA software (StataCorp LP, College Station, TX, USA).

\section{Results}

During the academic year 2010, there were 189 students who attended the EM rotation. Of those, 77 students (40.74\%) were in the traditional EM curriculum, while 112 students were in the new EM curriculum. Thirteen students (6.88\%) in the new EM curriculum were excluded from the repeated measures ANOVA due to incomplete evaluation forms.

There were no differences of sex and overall satisfaction between the traditional and new EM curriculum (Table 2). The average satisfaction score in teaching method of the new EM curriculum group was higher than the traditional EM curriculum group (4.54 versus [vs] 4.07, $P$-value $<0.001)$.

The top three highest average satisfaction scores in the new EM curriculum group were trauma workshop, bedside teaching, and EMS workshop. The mean (standard deviation) satisfaction scores of those three teaching methods were 4.70 (0.50), 4.63 (0.58), and $4.60(0.55)$, respectively. Satisfaction scores of all teaching methods are shown in Figure 1. Compared with other teaching methods, trauma workshop had a significantly higher satisfaction score than a traditional lecture, airway workshop, and EMS workshop (Figure 1). EMS workshop also had a significantly higher satisfaction score compared to airway workshop ( $P$-value 0.021$)$.

\section{Discussion}

Overall satisfaction was not different between the traditional and new EM curriculums. The satisfaction in teaching method of the new EM curriculum however was significantly higher than the traditional one (Table 2). The mean satisfaction score in teaching method increased from 4.07 to 4.54

Table 2 Male sex, overall satisfaction, and satisfaction with teaching methods by curriculum

\begin{tabular}{llll}
\hline Factors & $\begin{array}{l}\text { Traditional EM } \\
\text { curriculum } \\
\text { N=77 }\end{array}$ & $\begin{array}{l}\text { New EM } \\
\text { curriculum } \\
\mathbf{N}=\text { I I 2 }\end{array}$ & P-value \\
\hline Male sex, number (\%) & $31(40.26)$ & $43(38.39)$ & 0.880 \\
Overall satisfaction $^{\mathrm{a}}$ & $4.36(0.67)$ & $4.50(0.60)$ & 0.119 \\
Satisfaction in $^{\text {teaching method }}$ & $4.07(0.90)$ & $4.54(0.65)$ & $<0.00$ I \\
\hline
\end{tabular}

Notes: andicates the number of students was not equal to 77 or 112 in the traditional EM and new EM curriculum, respectively; data based on the 5 point Likert score and presented as mean (standard deviation) unless otherwise indicated. Abbreviation: EM, Emergency Medicine.
(11.55\% from the traditional curriculum). Improvement of satisfaction score in teaching methods of the new EM curriculum indicated that teaching with workshops increased student satisfaction in EM learning. A previous study also showed that teaching with active participation by students increased learning score and satisfaction.?

In the new EM curriculum, trauma workshop had the highest satisfaction score, followed by bedside teaching, EMS workshop, traditional lecture, and airway workshop, respectively. The trauma workshop also had a significantly higher satisfaction score compared with all other teaching methods (Figure 1). The trauma workshop simulated real situations and all students had their own role to play as a team member during the advanced cardiac life support session. This workshop therefore taught students real practical learning points. There were four important components in the trauma workshop that may be helpful in learning; simulation or real practice, group learning, immediate feedback, and problem-based or clinical thinking. ${ }^{7-13}$

The EMS workshop also had a significantly higher satisfaction score compared with airway workshop. The EMS workshop had unique objectives for EM curriculum. Students may gain new skills in emergency transfer system and methods which they had never experienced in any other departments. Airway workshop had the lowest satisfaction score. Students in the latter half of the year had passed almost all clerkships and may already have had experience regarding airway management from other departments such as anesthesiology, internal medicine, and surgery. Therefore, they may not gain any new knowledge. In addition, instructors spent most of the time teaching and demonstrating airway management and respiratory setup. Only a few students had a chance to practice. It may be worthwhile to let students practice more during the airway workshop. It should be noted that traditional lectures had a lower satisfaction score than other teaching methods except the airway workshop. Once again, teaching in a real situation or real patients in bedside teaching was preferred by clinical year medical students.

There are some limitations in this study. Due to short EM rotation for the fifth year medical students, clinical performance evaluations were not performed. Students were randomly allocated for the EM rotation by the academic affair unit but not randomly allocated to one of the EM curriculums. Further studies may be performed in randomized fashion with clinical performance evaluation and also in other university hospitals.

In conclusion, teaching EM with workshops improved student satisfaction in EM education for medical students. 


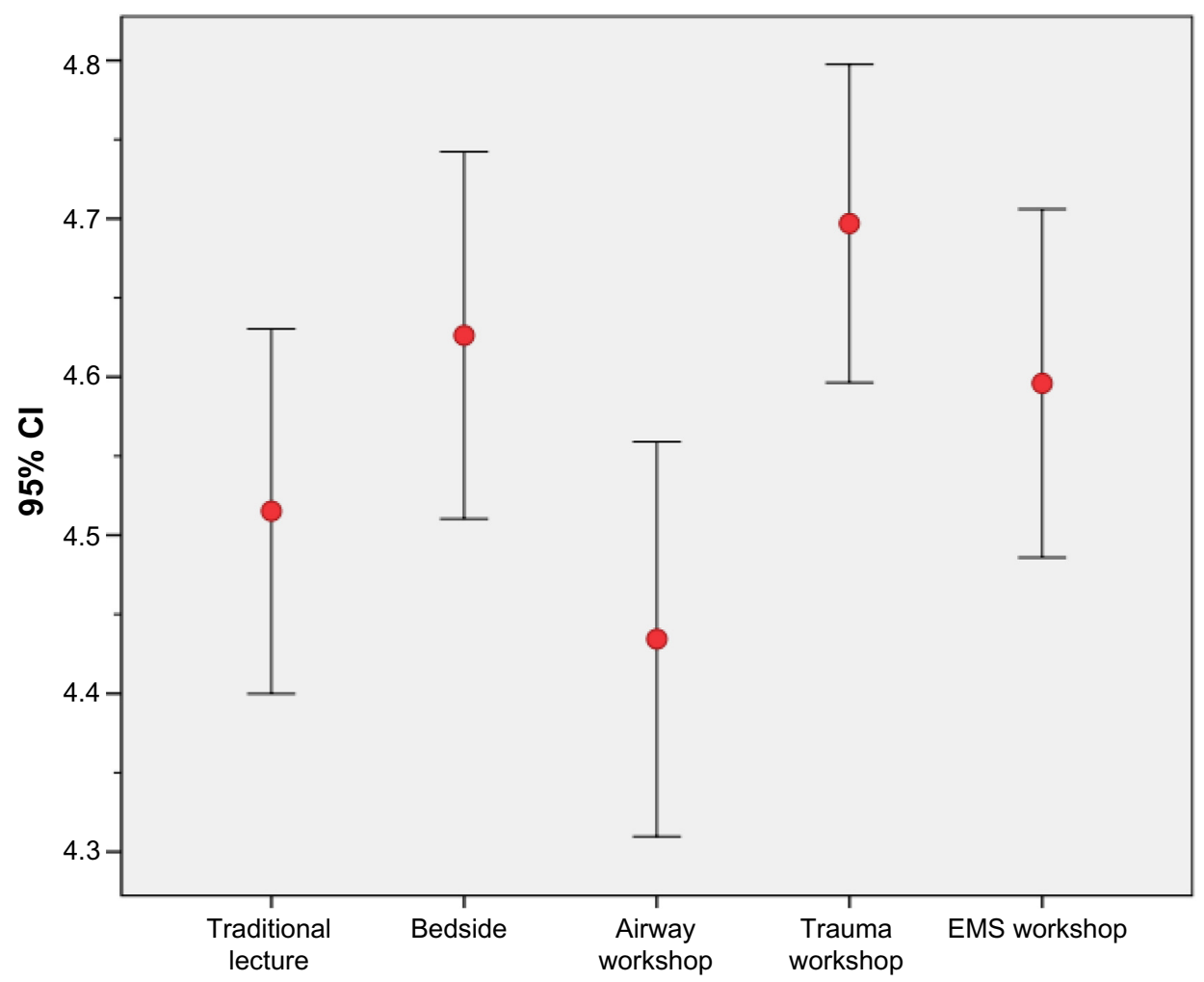

Figure I Satisfaction scores of all five teaching methods in the new Emergency Medicine curriculum.

Notes: Significant differences between teaching methods were trauma versus (vs) traditional (correlation coefficient -0 . I82; $P$-value 0.030 ); trauma vs airway (correlation coefficient $-0.260 ; P$-value $<0.001$ ); trauma vs EMS (correlation coefficient $0.100 ; P$-value 0.034 ); EMS and airway (correlation coefficient $-0.162 ; P$-value 0.021 ). Red dots indicate mean values; line indicates $95 \%$ confidence interval $(\mathrm{Cl})$.

Abbreviation: EMS, emergency medical services.

\section{Acknowledgments}

This study was supported by TRF grants from the Senior Research Scholar Grant, Thailand Research Fund grant number RTA5580004, and the Higher Education Research Promotion and National Research University Project of Thailand, Office of the Higher Education Commission, Thailand, through the Health Cluster (SHeP-GMS), Khon Kaen University. The authors gratefully thank Prof James A Will (University of Wisconsin, USA) for his kind review of the manuscript.

\section{Disclosure}

The authors report no conflicts of interest in this work.

\section{References}

1. Hurst JW. The overlecturing and underteaching of clinical medicine. Arch Intern Med. 2004;164(15):1605-1608.

2. Smith IK, Smith JO, Durand RP. Guidelines for planning faculty development workshops. J Biocommun. 1983;10(2):8-14.

3. Bland CJ, Reineke RA, Welch WW, Shahady EJ. Effectiveness of faculty development workshops in family medicine. J Fam Pract. 1979;9(3): 453-458.

4. LaCombe MA. On bedside teaching. Ann Intern Med. 1997;126(3): 217-220.
5. Ramani S, Orlander JD, Strunin L, Barber TW. Whither bedside teaching? A focus group study of clinical teachers. Acad Med. 2003;78(4):384-390.

6. Wilkerson L, Irby DM. Strategies for improving teaching practices: a comprehensive approach to faculty development. Acad Med. 1998;73(4):387-396.

7. Fatmi M, Hartling L, Hillier T, Campbell S, Oswald AE. The effectiveness of team-based learning on learning outcomes in health professions education: BEME Guide No 30. Med Teach. 2013;35(12):e1608-e1624.

8. Fisher JM, Walker RW. A new age approach to an age old problem: using simulation to teach geriatric medicine to medical students. Age Ageing. 2014;43(3):424-428.

9. Pratten MK, Merrick D, Burr SA. Group in-course assessment promotes cooperative learning and increases performance. Anat Sci Educ. 2014;7(3):224-233.

10. Syed Sheriff RJ, Bass N, Hughes P, et al. Use of interactive teaching techniques to introduce mental health training to medical schools in a resource poor setting. Afr $J$ Psychiatry (Johannesbg). 2013;16(4):256-263.

11. Meo SA. Evaluating learning among undergraduate medical students in schools with traditional and problem-based curricula. Adv Physiol Educ. 2013;37(3):249-253.

12. Yu D, Zhang Y, Xu Y, Wu J, Wang C. Improvement in critical thinking dispositions of undergraduate nursing students through problem-based learning: a crossover-experimental study. J Nurs Educ. 2013;52(10):574-581.

13. Medina MS, Conway SE, Davis-Maxwell TS, Webb R. The impact of problem-solving feedback on team-based learning case responses. Am J Pharm Educ. 2013;77(9):189. 
Advances in Medical Education and Practice

Dovepress

\section{Publish your work in this journal}

Advances in Medical Education and Practice is an international, peerreviewed, open access journal that aims to present and publish research on Medical Education covering medical, dental, nursing and allied health care professional education. The journal covers undergraduate education, postgraduate training and continuing medical education including emerging trends and innovative models linking education, research, and health care services. The manuscript management system is completely online and includes a very quick and fair peer-review system. Visit http://www.dovepress.com/testimonials.php to read real quotes from published authors.

Submit your manuscript here: http://www.dovepress.com/advances-in-medical-education-and-practice-journal 